George C. Zografos

Flora Zagouri

Theodoros N. Sergentanis

Afrodite Nonni

Nikolaos Lymperopoulos

Effstratios Patsouris

\title{
What parameters affect pain in core biopsy?
}

Dear Editor,

Core-needle biopsy with ultrasound guidance is a widely used and wellstudied method for the differential diagnosis of non-palpable breast lesions. However, the pain during the procedure is a rather neglected issue; in a recent issue of European Radiology, Hemmer et al. [1] provided an important insight into this topic, which affects a significant number of patients. Interestingly enough, they found a correlation between the number of passes, the depth of the lesion, the experience of the operator and the density of the breast. We have conducted a study in order to evaluate additional variables that could affect the pain experienced during the biopsy or which may prevent it.

Core biopsy (14-gauge) under ultrasound guidance was performed on 50 consecutive pre-menopausal women with a suspicious non-palpable solid breast lesion, BI-RADS 4, yielding a benign diagnosis. The women were aged 24-52 years old. All patients passed screening for mental impairment with the Mini-Mental-State Exam and for psychosis with the Schedule for Affective Disorders and Schizophrenia. No patient received drugs that could possibly modulate the intensity of the pain experience, such as non-steroid anti-inflammatory drugs, benzodiazepines, antidepressants, adrenocortical steroid synthetic analogues. The study was approved by the Review Board of the Institution. For the performance of the biopsy, $5 \mathrm{ml}$ of $1 \%$ lidocaine without epinephrine (superficially) and $10 \mathrm{ml}$ of $1 \%$ lidocaine (deeply) were administered. The visual analogue scale (VAS) was used to quantify the pain. VAS is a graphic rating scale (from 0 to 10 ) with a $10-\mathrm{cm}$ baseline, divided into ten equal parts from 0 (no pain) to 10 (worst possible pain), with a score of 5 (moderate pain) as the mid-point. At the end of the biopsy, the patients were asked to indicate the mean pain.

The patient's age, the history of breast feeding, the phase of menstrual cycle and the breast density (classified according to the American College of Radiology's breast imaging and reporting data system categories as follows: 1 , almost entirely fatty; 2 , scattered fibraglandular tissue; 3, heterogeneously dense; 4, extremely dense) were retrieved from the patient's chart review. Additionally, the duration of the biopsy was written down directly after the end of the procedure. The mean pain experienced (VAS score) was correlated.

The VAS score (mean $\pm \mathrm{SD}$ ) was $3.75 \pm 1.38$. Women who were at the luteal phase of their menstrual cycle $(n=16)$ exhibited marginally higher VAS score compared with women who were not $(n=20)$, i.e. $4.25 \pm 1.53$ vs $3.35 \pm 1.14(P=0.073$, Mann-WhitneyWilcoxon test for independent samples). History of breast feeding was not associated with VAS score $[3.93 \pm 1.60$ for women who had not breastfed $(n=$ 15) vs $3.62 \pm 1.24$ for women who had breastfed $(n=21) ; P=0.621$, Mann-

Fax: +30-210-6426390 
Whitney-Wilcoxon test for independent samples]. There was a positive association between VAS score and breast density (Spearman's $r h o=0.60$, $P<0.001)$.

In conclusion, pain during core biopsy under ultrasound may be influenced by a host of factors; some of them are immutable, inherent to the patient and others (phase of menstrual cycle) are variable. Avoidance of biopsy during the luteal phase may be one practical step in the minimisation of pain experienced.

Open Access This article is distributed under the terms of the Creative Commons Attribution Noncommercial License which permits any noncommercial use, distribution, and reproduction in any medium, provided the original author(s) and source are credited.

\section{References}

1. Hemmer JM, Kelder JC, van Heesewijk HP (2007) Stereotactic large-core needle breast biopsy: analysis of pain and discomfort related to the biopsy procedure. Eur Radiol Epub ahead of print. DOI 10.1007/s00330-007-0762-3 